Brief Original Article

\title{
Critical evaluation of antimicrobial use - A Turkish university hospital example
}

\author{
Salih Hosoglu ${ }^{1}$, Zafer Parlak ${ }^{1}$, Mehmet Faruk Geyik², Yilmaz Palanci ${ }^{3}$ \\ ${ }^{1}$ Dicle University, School of Medicine, Department of Infectious Diseases and Clinical Microbiology, Diyarbakir, \\ Turkey \\ ${ }^{2}$ Düzce University Hospital, Düzce, Turkey \\ ${ }^{3}$ Dicle University, School of Medicine, Department of Public Health, Diyarbakir, Turkey
}

\begin{abstract}
Introduction: Antimicrobials are being used unnecessarily for different reasons. The aims of this study were: assessment of the quality of antimicrobial use and determination of the factors related to correct use.

Method: Antimicrobial practice at Dicle University Hospital (DUH) was evaluated with a point prevalence approach. Using a standardized data collection form, the patients' data (clinic, epidemiology, laboratory and antimicrobial use) was collected. Possible influential factors on antimicrobial use were examined.

Results: In the surveillance study 1,350 inpatients were evaluated; 461 (34.1\%) of them were using antimicrobials for treatment and 187 $(13.9 \%)$ for prophylaxis. Antimicrobial indication was found in 355 of 461 patients $(77.0 \%)$, and the number of antimicrobials was 1.8 per patient in the treatment group. The most common reason for antimicrobial use was community-acquired infection (57.9\%). Pneumonia (20.4\%), skin and soft tissue infections (9.11\%) and urinary tract infections (7.9\%) were the most common infectious diseases. Positive culture results were available for 39 patients $(8.5 .0 \%)$ when antimicrobial treatment started. All steps of antimicrobial use were found appropriate in 243 patients $(52.7 \%)$.

In multivariate analyses, clinical manifestation of infection at the beginning $(p<0.001)$, presence of leukocyte counting $(p<0.001)$ and prescription by an infectious disease specialist were found significantly positive factors for wholly appropriate antimicrobial use. Hospitalization with a diagnosis other than infection was found a significantly negative factor for appropriate antimicrobial use ( $\mathrm{p}=0.001$ ).

Conclusion: The quality of antimicrobial use could be improved with better clinical and laboratory diagnosis and consultation with infectious diseases specialists
\end{abstract}

Key words: antimicrobial use; quality evaluation; relatedfactors

J Infect Dev Ctries 2013; 7(11):873-879. doi:10.3855/jidc.2921

(Received 06 August 2012 - Accepted 18 June 2013)

Copyright (C) 2013 Hosoglu et al. This is an open-access article distributed under the Creative Commons Attribution License, which permits unrestricted use, distribution, and reproduction in any medium, provided the original work is properly cited.

\section{Introduction}

Antimicrobials are being used unnecessarily and with inappropriate characteristics, for different reasons. Thus, antimicrobial resistance has become one of the most important issues over the last 20 years. Many studies have focused on the influential factors of antimicrobial use, as well as aiming to determine the criteria for optimal quality of antimicrobial therapy [13]. It is generally accepted that the ideal antimicrobial use should be combined with maximal efficacy, minimal toxicity and the lowest cost. The quality of antimicrobial prescriptions depends on multiple factors. One of the most important factors is the knowledge of its prescribers. In the prescription of antimicrobials, the host factors, the virulence of microorganisms, the pharmacokinetics and the pharmacodynamics of drugs must be considered [1,49].

Antimicrobial resistance and excessive antimicrobial use are important issues in Turkey. The total annual expenditure for antimicrobials in Turkey in 2002 was $24 \%$ of all drug spending. Conversely, some studies revealed that the quality of antimicrobial use in Turkey is not satisfactory. A new restriction on antimicrobials was implemented as a method to improve antimicrobial drug use by May 2003. After May 2003, the reimbursement of wide-spectrum antimicrobials was cut, unless it was approved by an Infectious Diseases Physician (IDP) [10].

We evaluated antimicrobial practices at Dicle University Hospital (DUH) with an observational approach. This study evaluated the necessity and quality of antimicrobial drug prescribing. In the 
present study, we aimed to assess the quality of antimicrobial use and to determine the factors related to correct use of antimicrobials in patients.

\section{Methodology}

Dicle University Hospital (DUH) is a referral hospital in the centre of Diyarbakir city. The hospital is the largest teaching hospital, with 1146 beds, in southeast Turkey. The hospital comprises departments covering all major specialties. In 2006, a total of 42,843 patients were hospitalized. The occupation rate of hospital beds was $64 \%$ in 2006 .

\section{Study design}

From a prospective approach, the administration of antimicrobials was examined in all inpatients at the hospital, for which they were prescribed during the study days, 4 May 2006 and 4 October 2006. The epidemiological and medical data of all hospitalized patients was reviewed during the study days. The indication of antimicrobial use, the substance prescribed, the duration of treatment, the dosage, the route and intervals of antimicrobial administration were also recorded. The evaluation of the necessity of antimicrobials from a prospective approach was carried out through a point scoring system as a quality indicator; prophylactic use of antimicrobials was recorded on the same form, but this data will be evaluated elsewhere. The data was collected by a research team from the Department of Infectious Diseases and all cases were evaluated by specialists from the same department. The final decision on antimicrobial use was given by IDPs (S.H. and M.F.G). The appropriateness of antimicrobial treatment was evaluated using modified Kunin's criteria [6].

The following categories were used:

I. The use of antimicrobial therapy is necessary; the protocol (choice, route, duration, and dosage) is appropriate. This decision may have been supported with a microbiology report.

II. The use of antimicrobial therapy is necessary, but a different antimicrobial (less expensive, less toxic, or another combination) is preferred.

III. The use of antimicrobial therapy is necessary, but a different antimicrobial dose, interval, duration or route of administration is preferred.

IV. Disagreement with the use of antimicrobial therapy; administration is unjustified. Category I indicates "appropriate therapy," Categories II and III indicate that there was some major deficiency in the choice or use of antimicrobials. Category IV indicates unnecessary antimicrobial use. The evaluation and categorization of antimicrobial treatment was carried out based on the literature and clinical experiences of the authors [6-9,11-22].

Possible influencing factors on the quality of antimicrobial use were examined. At this stage, the cases in Category I were entered into the model as appropriate antimicrobial use and the others were labeled inappropriate.

\section{Statistical analyses}

The quality of antimicrobial use was evaluated for association with the following predictor variables: type of department (surgical or medical), type of health insurance, primary hospitalization reason (emergency, elective surgical, medical, infections), severity of the disease, fever $\left(>38{ }^{0} \mathrm{C}\right)$, existence of a leukocyte increase, neutrophily $(>70 \%)$, existence of a Creactive protein test, increasing erythrocyte sedimentation rate (ESR) $(>30 \mathrm{~mm} / \mathrm{h})$ and decisionmaker for antimicrobial use (resident from same department, academic from same department, IDP). The data was analyzed using SPSS version 10.0 (SPSS Inc, Chicago, IL). The $\chi^{2}$ test and independent sample t-tests were used to assess the strength of the association between variables. Multiple logistic regression modeling was performed to identify predictor variables that influenced the quality of antimicrobial use.

\section{Results}

In the surveillance study, the first surveillance was started on 4 May 2006 and the second started on 4 October 2006. In total, 1,350 inpatients were evaluated, with 461 of them (34.1\%) using antimicrobial for treatment and $187(13.9 \%)$ for prophylaxis. The occupancy rate of the hospital was $58.9 \%$ during the study days. In total, 648 patients were using antimicrobials $(48.0 \%)$. Antimicrobial use for prophylaxis was very rare in medical departments but more common in surgical departments $(0.3 \%$ vs. $28.2 \%$, respectively). In total, 364 of $656(55.4 \%)$ patients were using antimicrobials in surgical departments and 284 of $694(40.9 \%)$ patients in internal departments $(\mathrm{p}=0.0016)$. Antimicrobial use for treatment was more common in medical departments (282 patients, 40.6\%) than in surgical departments (179 patients, $27.3 \%) \quad(\mathrm{p}<0.001)$. The route of antimicrobial use was intravenous in 556 cases $(73.5 \%)$, oral in 198 cases $(26.1 \%)$ and intramuscular in three cases $(0.4 \%)$. 
Assessment of antimicrobial use in the treatment group

The mean age of patients was $31.5( \pm 25.4)$ (range $0-80$ years), and $255(55.3 \%)$ of them were male. In the treatment group, the most common reason for hospitalization was (non-infectious) internal disease (174 patients, 37.7\%) (Table 1). The most common reason for antimicrobial use was community-acquired infection (267 patients, 57.9\%) (Table 2). The most common infectious diseases were: pneumonia (94 patients, 20.4\%), skin and soft tissue infections (43 patients, $9.11 \%$ and urinary tract infections (36 patients, 7.9\%). The most commonly used antimicrobials were ceftriaxone (124 cases, 26.9\%), ampicilline-sulbactam (59 cases, 12.8\%) cefazoline (45 cases, 9.8\%), and meropenem (45 cases, 9.8\%). Antimicrobial treatment was arranged according to positive culture results in 39 patients $(8.5 .0 \%)$.

The decision for antimicrobial treatment was made by responsible academics from the same department in 347 cases $(75.3 \%)$, by resident physicians in 24 cases (5.2\%), and by infectious diseases physicians/consultants in 78 cases (16.9\%). In 12 cases $(2.6 \%)$, responsible academics/physicians added one or more antimicrobials after IDP consultations. All of these 12 cases had been treated with unnecessary antimicrobials. The mean number of antimicrobials was 1.8 per patient in the treatment group.

\section{Assessment of antimicrobial use}

Antimicrobial use was found necessary in 355 patients $(77.0 \%)$ in the treatment group. In 243 (52.7\%) patients, all steps of antimicrobial use were found appropriate (Category I). In 74 patients (16.1\%), antimicrobials were needed, but the choice of antimicrobial treatment was inappropriate (Category II). In 53 patients $(11.5 \%)$, the inappropriateness was having used two antimicrobials that had similar spectrums, and in 21 patients $(4.6 \%)$ the spectrum of antimicrobials was broader than the spectrum needed. In 40 patients $(8.7 \%)$, the antimicrobial treatment was necessary, but the treatment was not acceptable (Category III). In these patients, the following types of inappropriateness were found: narrower spectrum than needed (24 cases, 5.2\%), not given the necessary combination (6 patients, 1.3\%), wrong choice and wrong dosage (5 patients, 1.1\%), unnecessary toxic antimicrobials ( 3 patients, 0.7\%), and wrong choice and wrong interval ( 2 patients, $0.4 \%$ ). There was no indication to use antimicrobials in 106 patients $(23.0 \%)$ (Category IV).
The related factors of quality of antimicrobial use

The factors related to appropriate antimicrobial use were evaluated. In the univariate analyses, primary hospitalization reason, critical situation upon admission, fever, clinical manifestation of infection at admission, existence of a leukocyte increase, neutrophil elevation, existence of a C-reactive protein test and decision-maker for antimicrobial use were found significant factors in appropriate antimicrobial use (Table 3).

In the multivariate analyses, clinical manifestation of infection at the beginning $(\mathrm{OR}=3.8 ; \mathrm{CI}=1.9-5.4$; $\mathrm{p}<0.001)$, existence of a leukocyte increase $(\mathrm{OR}=3.3$; $\mathrm{CI}=1.2-9.2 ; \mathrm{p}<0.001)$ and antimicrobial decision made by an IDP $(\mathrm{OR}=7.4 ; \mathrm{CI}=3.5-15.3 ; \mathrm{p}<0.001)$ were considered significantly positive factors for appropriate antimicrobial use. Hospitalization with a medical reason other than infection $(\mathrm{OR}=0.5$; $\mathrm{IR}=0.3$ $0.7 ; \mathrm{p}=0.001$ ) was found as a significant negative factor for appropriate antimicrobial use.

\section{Discussion}

Many studies have shown that more than one-third of antimicrobials prescribed for hospitalized patients are not necessary [7-9,20,21,26]. Antimicrobial stewardship programs constitute a basis for prevention strategies to control the emergence and spread of antimicrobial-resistant pathogens in hospitals alongside effective infection control and prevention measures. Antimicrobial resistance has become one of the most difficult problems in medical treatment in the world. Many studies have been implemented to evaluate and control resistance processes. Unfortunately, none of them have discovered a clear solution to stop the resistance. Most of them have focused on the quantity of antimicrobial use $[6,11]$.

The quality of antimicrobial use in hospital settings has been evaluated in few studies. The quality of antimicrobial use may be primarily evaluated based on the following criteria: reasonable indication, correct drug choice with maximal efficacy, minimal toxicity at the lowest cost, correct dosage interval and correct route. The acceptable indication should be supplemented with a suitable antimicrobial treatment course $[7,8]$. This study has concentrated on issues of the quality of antimicrobial drug use for hospitalized patients and possible influential factors of appropriate antimicrobial use. In this study, Kunin's criteria were modified and used in a simpler way [6-9]. 
Table 1: Reasons for hospitalization of 461 patients

\begin{tabular}{lc}
\hline Hospitalization reasons & No. of patients (\%) \\
\hline Medical disorders (non-infectious) & $174(37.7)$ \\
Community acquired infections & $119(26.8)$ \\
Elective surgical intervention & $41(8.9)$ \\
Emergent surgical intervention & $35(7.6)$ \\
Multiple disorders & $33(7.2)$ \\
Medical + surgical reasons & $30(6.5)$ \\
Nosocomial infections & $20(4.3)$ \\
Burn & $9(2.0)$ \\
Total & $\mathbf{4 6 1 ( \mathbf { 1 0 0 } )}$ \\
\hline
\end{tabular}

Table 2: Indications of antibiotic use of 461 patients

\begin{tabular}{lc}
\hline Reasons for antibiotic use & No. of patients (\%) \\
\hline Community acquired infections (empirical) & $237(51.4)$ \\
Nosocomial infections (empirical) & $77(16.7)$ \\
Nosocomial infections (microbiologic evidence) & $39(8.5)$ \\
Community acquired infections (microbiologic evidence) & $30(6.5)$ \\
Community acquired + Nosocomial infections & $3(0.7)$ \\
Unknown & $75(16.3)$ \\
Total & $\mathbf{4 6 1 ( \mathbf { 1 0 0 } )}$ \\
\hline
\end{tabular}

Table 3 - Univariate analyses of related factors with appropriate antimicrobial use

\begin{tabular}{|c|c|c|c|c|c|}
\hline Variables & Appropriate & Inappropriate & OR & $\% 95 \mathrm{CI}$ & P value \\
\hline Departments (Surgical) & $78 / 243$ & $84 / 218$ & 0.747 & $0.509-1.097$ & 0.137 \\
\hline \multicolumn{6}{|l|}{ Type of insurance } \\
\hline Low income group (government support) & $140 / 243$ & $119 / 218$ & 1.119 & $0.773-1.620$ & 0.552 \\
\hline Workers/professional & $64 / 243$ & $69 / 218$ & 0.766 & $0.511-1.148$ & 0.196 \\
\hline Governmental workers & $21 / 243$ & $18 / 218$ & 1.045 & $0.541-2.019$ & 0.895 \\
\hline Other & $16 / 243$ & $10 / 218$ & 1.458 & $0.647-3.286$ & 0.360 \\
\hline \multicolumn{6}{|l|}{ Primary hospitalization reason } \\
\hline Emergent surgical & $26 / 243$ & $17 / 218$ & 1.416 & $0.746-2.687$ & 0.286 \\
\hline Elective surgical & $25 / 243$ & $36 / 218$ & 0.576 & 0.333-.996 & 0.046 \\
\hline Non-infectious & $97 / 243$ & $136 / 218$ & 0.396 & $0.272-.578$ & $<0.001$ \\
\hline Infectious & $123 / 243$ & $59 / 218$ & 2.774 & $1.875-4.103$ & $<0.001$ \\
\hline Critical situations upon admission & $123 / 243$ & $44 / 218$ & 4.040 & $2.665-6.126$ & $<0.001$ \\
\hline Fever $\left(>38{ }^{0} \mathrm{C}\right)$ & $122 / 237$ & $58 / 206$ & 2.707 & $1.821-4.024$ & $<0.001$ \\
\hline Clinical manifestation of infections & $214 / 242$ & $117 / 218$ & 6.707 & $4.144-10.855$ & $<0.001$ \\
\hline Leukocyte increase & $235 / 243$ & $193 / 218$ & 4.001 & $1.681-9.523$ & 0.001 \\
\hline PMNL $(>70 \%)$ & $147 / 235$ & $92 / 198$ & 1.855 & $1.259-2.733$ & 0.002 \\
\hline CRP measurement & $165 / 243$ & $121 / 218$ & 1.682 & $1.149-2.463$ & 0.007 \\
\hline ESR increased $(>30 \mathrm{~mm} / \mathrm{h})$ & $77 / 126$ & $49 / 101$ & 1.668 & $0.982-2.832$ & 0.058 \\
\hline \multicolumn{6}{|l|}{ Decision maker of treatment: } \\
\hline Resident from department & $13 / 243$ & $13 / 218$ & 0.886 & $0.402-1.956$ & 0.765 \\
\hline Academic from department & $151 / 243$ & $193 / 218$ & 0.361 & $0.236-0.551$ & $<0.001$ \\
\hline$I D P$ & $78 / 243$ & $10 / 218$ & 9.798 & $4.916-19.525$ & $<0.001$ \\
\hline
\end{tabular}


Antimicrobials consist of a significant part of drugs prescribed in hospital settings. In Turkey, in contrast with many other countries, antimicrobials are the drugs that are used first $[5,26,27]$. The proportion of antimicrobial use reported in hospitalized patients in Croatia was 15\% (Department of Medicine), 44.5\% in Thailand (university hospital), (30.6\% in Turkey (multi-central) and $88.8 \%$ in China [7,27-29]. In our study, the proportion was $48 \%$. These proportions could be related to different variables, such as the patients' characteristics. Some studies reported that antimicrobial use was more common in surgical departments than in medical departments. This was concordant with our study when prophylactic antimicrobial uses were included $[28,30]$.

The proportion of inappropriate antimicrobial use was reported in a wide range. The variation could be related to the evaluation methods for antimicrobial use. At the same time, the patients' characteristics are important. Some studies included only treatment purposes, but some of them included treatment and prophylactic purposes together. In this study, the duration of treatment was not evaluated because of the study design. A multi-centre study from Turkey reported that $61.5 \%$ of prescriptions were evaluated as clinically appropriate in patients receiving antimicrobials for treatment [27]. Another study reported $45.7 \%$ appropriate use in a hospital setting [30]. In this study, the appropriate proportion was found as $52.2 \%$. These studies have revealed that at least half of antimicrobial prescriptions at Turkish hospitals could be regarded as inappropriate.

Prescription without antimicrobials is one of the most frequent characteristics of inappropriate treatments. In the present study, according to the prescribers' statements, $16.3 \%$ of the prescriptions had no reason for antimicrobial use. On the other hand, in our assessment, $23.0 \%$ of the antimicrobial use was unnecessary. Vlahović-Palčevski et al. reported the same proportion $(23 \%)$ for unnecessary antimicrobial prescriptions in Croatia [9]. They reported that the main reason for inappropriate antimicrobial treatment was the wrong choice of antimicrobials (37\%).

The quality of antimicrobial prescription is related to the accuracy of the diagnosis of the infection. A Norwegian study reported that bacteriological samples were obtained from $85 \%$ of the patients and compliance with the guidelines was $>90 \%$. Compliance was highest when the results of bacteriological samples were positive [31]. In Rijeka Hospital, $32 \%$ of the antimicrobial prescriptions were based on microbiology results [8]. In our study, only
$8.5 \%$ of prescriptions were based on microbiological evidence. The majority of the prescriptions were arranged empirically. Physicians frequently chose broad-spectrum antimicrobials because of diagnostic uncertainty. Limited use of the microbiology laboratory in the decision for antimicrobial prescriptions could be a reason for the low quality of antimicrobial use. Improving adherence to local or major guidelines for antimicrobial prescription could be another effective approach to decrease inappropriate antimicrobial use.

In the multivariate analyses, clinical manifestation of infection at the beginning was a supporting factor for appropriate antimicrobial use. The existence of clinical manifestation makes it easier to accurately diagnose the infection. Effective use of laboratory methods other than microbiology (leukocytes, Creactive protein) could be helpful for accurate diagnosis of infections. In particular, an increasing number of leukocytes/neutrophils could be a valuable marker for acute infections. In our study, the existence of a leukocyte increase was found as one of the related factors for appropriate antimicrobial prescriptions. These data indicate that some doctors do not use simple and basic laboratory tools for confirmation of infection.

The debate on the decision-makers for antimicrobials is ongoing. Many studies have indicated that IDPs with a multidisciplinary team approach play a critical role in controlling antimicrobial use in the hospital $[12,26,32]$. Our study confirmed these data, and showed that antimicrobial decisions made by an IDP were a significantly positive factor for appropriate antimicrobial use.

This study provided many new data on the behaviours of antimicrobial prescriptions in a Turkish university hospital. On the other hand, this study has some limitations. The study was organized and performed by infectious diseases physicians. This situation could be considered a risk for bias. A specialist from the public health department monitored the study to avoid such risks.

\section{Conclusion}

In conclusion, almost half of the hospitalized patients were prescribed antimicrobial drugs in this study. Nearly the same proportion of antimicrobial prescriptions for treatment was found as inappropriate. The quality of antimicrobial use could be improved with better clinical and laboratory diagnoses and consultation with infectious diseases specialists. 
Further studies are needed to define the most efficient approaches to improve hospital antimicrobial drug use.

\section{References}

1. Charani E, Cooke J, Holmes A (2010) Antibiotic stewardship programmes-what's missing? J Antimicrob Chemother 65: 2275-2277.

2. Cooke D, Salter AJ, Phillips I (1980) Antimicrobial misuse, antibiotic policies and information resources. J Antimicrob Chemother 6:435-443.

3. McGowan JE (1983) Antimicrobial resistance in hospital organisms and its relation to antibiotic use. Rev Infect Dis 5: 1033-1048.

4. Apisarnthanarak A, Danchaivijitr S, Bailey TC, Fraser VJ (2006) Inappropriate antibiotic use in a tertiary care center in Thailand: an incidence study and review of experience in Thailand. Infect Control Hosp Epidemiol 27: 416-420.

5. Jacob JT and Gaynes RP (2010) Emerging trends in antibiotic use in US hospitals: quality, quantification and stewardship. Expert Rev Anti Infect Ther 8: 893-902.

6. Kunin CM, Tupasi T, Craig WA (1973) Use of antibiotics: A brief exposition of the problem and some tentative solutions. Ann Intern Med 79: 555-60.

7. Vlahovic-Palcevski V, Francetic I, Palcevski G, Novak S, Bergman U (2005) Antimicrobial prescribing at a university hospital: justified or 'just in case': testing a new scoring system as a key quality indicator. Pharmacoepidemiol Drug Saf 14: 561-66.

8. Gyssens IC (2001) Quality measures of antimicrobial drug use. Int J Antimicrobial Agents 17: 9-19.

9. Vlahovic-Palcevski V, Francetic I, Palcevski G, Novak S, Abram M, Bergman U (2007) Antimicrobial use at a university hospital: appropriate or misused? A qualitative study. Int J Clin Pharmacol Ther 45: 169-174.

10. Hoşoğlu S, Esen S, Ozturk R, Altindis M, Ertek M, Kaygusuz S, Caylan R, Demirdag K, Sencan I, Ertem GT, Aslan S, Bosnak V, Aygun P, Erol S, Celen MK (2005) The effect of a restriction policy on the antimicrobial consumption in Turkey: a country-wide study._Eur J Clin Pharmacol 61: 727-731.

11. Thuong M, Shortgen F, Zazempa V, Girou E, Soussy CJ, Brun-Buisson C (2000) Appropriate use of restricted antimicrobial agents in hospitals: the importance of empirical therapy and assisted re-evaluation. J Antimicrob Chemother 46: 501-508.

12. John JF Jr, Fishman NO (1997) Programmatic role of the infectious diseases physician in controlling antimicrobial costs in the hospital. Clin Infect Dis 24: 471-485.

13. Baum ML, Anish DS, Chalmers TC, Sacks HS, Smith H Jr, Fagerstrom RM (1981) A survey of clinical trials of antibiotic prophylaxis in colon surgery: evidence against further use of no-treatment controls. N Engl J Med 305: 795-799.

14. Shales DM, Gerding DN, John JF Jr, Craig WA, Bornstein DL, Duncan RA, Eckman MR, Farrer WE, Greene WH, Lorian V, Levy S, McGowan JE Jr, Paul SR, Ruskin J, Tenover FC, Watanakunakorn C (1997) Society for Healthcare Epidemiology of America and Infectious Diseases Society of America Joint Committee on the prevention of antimicrobial resistance in hospitals: guidelines for the prevention of antimicrobial resistance in the hospitals. Clin Infect Dis 25: 584-599.
15. LCDD Report (1990) Guidelines for antimicrobial utilization in health care facilities. J Infect Dis 1: 64-70.

16. Inan A, Daglı Ö, Akçay SS, Engin DO, Karagül E, Özyürek SC (2011) Antibiotic use and cost in a teaching hospital in Istanbul J Microbiol Infect Dis 1: 128-133.

17. Hartmann B, Junger A, Brammen D, Röhring R, Klasen J, Quinzio L, Benson M, Hempelmann G (2004) Review of antibiotic drug use in a surgical ICU: anagement with a patient data management system for additional outcome analysis in patients staying more than 24 hours. Clin Ther 26: 915-924.

18. Jones SR, Pannell J, Barks J, Bratton T, Yanchick YA, Bratton T, Browne R, McRee E, Smith JW (1977) The effect of an educational program upon hospital antibiotic use. Am J Med Sciences 273: 79-85.

19. Marr JJ, Moffet HL, Kunin CM (1988) Guidelines for improving the use of antimicrobial agents in hospitals: a statement by the Infectious Diseases Society of America. J Infect Dis 157: 869-876.

20. Woodward RS, Medoff G, Smith MD, Gray JL (1987) Antibiotic cost saving from formulary restrictions and physician monitoring in a medical-school-affiliated hospital. Am J Med 83: 817-823.

21. Hirschman SZ, Meyers BR, Bradbury K, Mehl B, Gendelman S, Kimelblatt B (1988) Use of antimicrobial agents in a university teaching hospital. Evolution of a comprehensive control program. Arch Intern Med 148: 2001-2007.

22. Pestotnik SL, Classen DC, Evans RS, Burke JP (1996) Implementing antibiotic practice guidelines through computer-assisted decision-support: clinical and financial outcomes. Ann Intern Med 124: 884-890.

23. Evans RS, Pestotnik SL, Classen DC, Clemmer TP, Weaver LK, Orme JF Jr, Lloyd JF, Burke JP (1998) A computerassisted management program for antibiotics and other antiinfective agents. New Engl J Med 338: 232-238

24. Wilkowske CJ (1991) General principles of antimicrobial therapy. Mayo Clin Proc 66: 931-941.

25. Hecker MT, Aron DC, Patel NP, Lehmann MK, Donskey CJ (2003) Unnecessary use of antimicrobials in hospitalized patients: current patterns of misuse with an emphasis on antianaerobic spectrum of activity. Arch Intern Med 163: 972978.

26. Erbay A, Çolpan A, Bodur H, Çevik MA, Samore MH, Ergönül Ö (2003) Evaluation of antibiotic use in a hospital with an antibiotic restriction policy. Int J Antimicrob Agents 21: 308-312.

27. Usluer G, Ozgunes I, Leblebicioğlu $H$ and the Turkish Antibiotic Utilization Study Group (2005) A multicenter point-prevalence study: antimicrobial prescription frequencies in hospitalized patients in Turkey. Ann Clin Microbiol Antimicrobial 4: 16-21.

28. Aswapokee N, Vaithayapichet S, Heller RF (1990) Pattern of antibiotic use in medical wards of a university hospital, Bangkok, Thailand. Rev Infect Dis 12: 136-141.

29. Hu S, Liu X, Peng Y (2003) Assessment of antibiotic prescription in hospitalized patients at a Chinese university hospital. J Hosp Infect 46: 161-163.

30. Pien FD, Lau WK, Sur N (1979) Antibiotic use in a small community hospital. West J Med 130: 498-502.

31. Berild D, Ringertz SH, Lelek M (2002) Appropriate antibiotic use according to diagnoses and bacteriological findings: report of 12 point-prevalence studies on antibiotic use in a university hospital. Scand J Infect Dis 34: 56-60. 
32. Tünger Ö, Dinç G, Özbakkaloğlu B, Atman UC, Algun U (2000) Evaluation of rational antibiotic use. Int J Antimicrob Agents 15: 131-5.

33. Nathwani D, Davey P (1999) Antibiotic prescribing - are there lessons for physicians? Q J Med 92: 287-292.

\section{Corresponding author}

Professor Salih Hoşoğlu

Dicle University Hospital

Department of Infectious Diseases and Clinical Microbiology

Diyarbakir, Turkey

Fax: +90 4122488440

E-mail: hosoglu@hotmail.com

Conflict of interests: No conflict of interests is declared. 